\title{
A Comprehensive Survey of Feature Extraction and Feature Selection Techniques of Face Recognition System
}

\author{
Swati Saini ${ }^{1}$, Dr. Preeti Malhotra ${ }^{2}$ \\ (mail to:sainiswati39@gmail.com¹,preetianeja2005@gmail.com²) \\ Student, Computer Science and Engineering Department, Hindu College of Engineering, Sonepat, Haryana, India ${ }^{1}$, \\ Assistant Professor, Computer Science and Engineering Department, Hindu College of Engineering, Sonepat, Haryana, India ${ }^{2}$
}

\begin{abstract}
Among various types of biometric techniques it is the face recognition that gains huge popularity in the last few decades due to its vast applicationsand more user friendly nature.Face Recognition is the procedure of identification and verification of a person's identity using his face. Main steps involved in a FR System are Face Detection, Feature Extraction, Feature Selection and Recognition. Feature Extraction and Feature Selection are the two main phases to be focused on in order to get a good FR System. In this paper, we have carried out anextensive literature review on face recognition focusing on feature extraction and feature selection phases a bit more.
\end{abstract}

Keyword:Face recognition (FR), feature extraction, principal component analysis (PCA), feature selection.

\section{Introduction}

Biometrics is the metrics related to human characteristics. Biometrics identify humans using their two types of characteristic namely- physiological and behavioral. Physiological traits involves face, finger, retina, DNA, etc. whereas behavioral traits involves gait, signature, keystroke, etc.[1]. Among all the above mentioned biometrics, it is the face recognition which has been the most fascinating topic for researchers since the past few decades being it convenient, more users friendly and an inexpensive technique of identification. The main phases of an FR System are: Face Detection, Feature Extraction, Feature Selection and Face Recognition. In the Face Detection phase, a human face is detected from the captured image/video. After detection, features are extracted from the captured face in feature extraction phase and only relevant features are selected from all the extracted features in feature selection phase in order to get much accurate results. Then comes the Face Recognition phase in whichthe captured face is compared with the models already present in database and recognition is done.The goodness of an FR System depends majorly on Feature Extraction and Feature Selection phase. To combine the process of Face Recognitionaltogether we can say, in an FRSystem, a new face is given to the system and is compared with the face 
templates/models already stored in the database and then is classified as a known individual if a correspondence is found [2].

Following this introduction is Section 2 presenting basics of anFR System including the brief introduction to various phases of a FRS. Then, Section 3 and Section 4 respectively discuss the basics and techniques of Feature Extraction and Feature Selection phasealong with their literature review. Section 5 represents results inferred from literature survey andfinally paper concludes in Section 6.

\section{Face Recognition System}

AnFR Systeminvolves various steps shown in Figure 1. In this, an image or a video is fed as an inputwhich is then pre-processed in order to improve quality of the input data. After that face is cropped from the whole image and background is removedin order to detect the face which is then followed by the Feature Extraction and Feature Selection phasein which the facial features are first extracted and then the most relevant ones are chosen. In the last phasei.e. Face Recognition phase, matching is done with a help of a templates generated for enrollment in the FRS for verification/identification of a particular person [3]. Verification answers "Is the person who they say they are?" whereas Identification give answer to "Who is this person?".

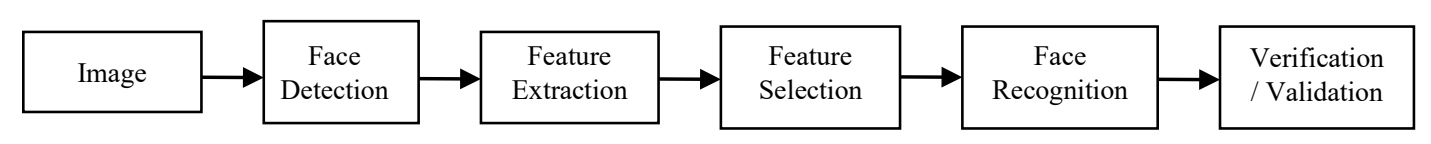

Figure 1: Key steps involved in Face Recognition Process(4)

The primary purpose ofFace Detection phase is to check whether human faces are present in the input image/video or not; if yes, then what is the location of those faces. The survey written by Wei-Lun Chao [4] classified face detection algorithms in four categories, namely: Knowledge-based methods, Feature Invariant Approach, Template Matching and Appearance-based Methods.Knowledge-based methods[5]are the rule based methods and deduce the relationship between facial features using some pre-defined rules regarding facial features and their relationship with each other. They use the human knowledge to determine how human face actually looks like. Algorithms following Feature Invariant approach[6] focuses on to look for structural features even in bad illumination conditions and pose variations and then locate faces in the input image using those features. In the Template Matching[7]methods category, various standard patterns of human faces (either whole or partial) are already stored in the system and then the input image is matched with those patterns to detect whether a human face is present in the input image or not.The training images which capture the representative variability of facial appearance are used to determine models which are then used to detect faces in Appearance-based methods [8].Face Recognition is extensively getting used in various sectors be it security-law enforcement, health or marketing \& retail. Face Recognition is cheaper, restricts people involvement, convenient and less time consuming. 'Illumination' and the 'pose variation' are two most common issues present in face recognition. Same pictures appear to be a different picture under several 
illumination effects. The functioning of anFR System degrades when large pose variations are present in the input images [9].

\section{Feature Extraction}

After detecting face in an image, the next step is to extract useful features only from the input image. Feature extraction is done to get rid of superfluous and beside the point features from the input image. The methods used to extract useful feature are divided into two types namely, Holistic Matching methods and Feature-based matching methods. The HolisticMatching Methods [10] or Appearance-based methods use entire face as an input to a recognition system whereas in the Geometric Feature[11] based Matching Methods the location of different features such as eyes, mouth etc. are first extracted and then fed as an input to anFRSystem [9]. Some very popular feature extraction techniques are:

\subsection{Principal Component Analysis (PCA)}

Karhunen-Loeve method [2] is an another name for PCA and is one of the most popular methods for solving the problem of overfitting. PCA is a dimension reduction technique with the goal to extract the most relevant information contained in the image. This reduced data is then used for recognition purpose[12]. A face contains certain set of features which are known as either the principal components or the Eigenfaces[13]. In PCA based face recognition, increasingeigen value is directly proportional to the recognition rate but the recognition rate become stagnant after a certain increase in the eigen value[12]. It is an unsupervised learning algorithm.

Various methods for face recognition are present in the literature such as Gabor Features[14], Diagonal Principal Component Analysis (DiaPCA)[15], PCA, ICA, LDA[16], a combination of PCA and LDA (PCA-LDA)[17][18]. Peng et al.[14] in 2004 carried out face recognition experiment on FERET database using Ada-Boosted Gabor Features and results showed that the rank 1 recognition rate improved to $95.2 \%$ from $37.5 \%$ when 700 features were selected against 6 features; also when re-sampling strategy was applied rank 1 recognition rate shoots to $92.8 \%$ (1740 features) from 90.6\% (700 features). The Gabor Feature method was then combined with PCA and KPCA (Kernel PCA) by Vinay et al.[19] in 2015 and the results proved that on Euclidean distance measure, the linear Gabor-PCA outperformed the non-linear Gabor-KPCA by $6.67 \%, 4.17 \%$ using MAHCOS, $0.83 \%$ on the cosine measure and $12.00 \%$ using the City-Block distance measure.

PCA being the simplest of all techniques is widely used for the dimension reduction process. However various new methods were proposed that were not just the extension of widely used PCA but also proved their superiority over PCA. Daoqiang et al. [15]in 2006 presented diagonal principal component analysis (DiaPCA) which as compare to standard PCA looks for best projective vectors from diagonal face images without image-to-vector transformation. DiaPCA $(90.50 \%$ recognition accuracy) performed better than both PCA (85.50\% accuracy) and 2DPCA (85.50\% accuracy) whereas the performance of DiaPCA further improved when it was combined with 2DPCA(DiaPCA $+2 \mathrm{DPCA}=91.50 \%$ accuracy).

To solve the memory requirement burden and computational cost issues of existing PCA, Haitao et al. [20]in 2006 proposed a method named Incremental PCA (IPCA) whose average recognition rate difference with PCA was less than $1 \%$. 


\subsection{Linear Discriminant Analysis (LDA)}

LDA is a supervised learning algorithm for dimension reduction which tries to preserve class discrimination information as much as possible. In case where number of samples per class are less, PCA performs better, whereas with large datasets having multiple classes, LDA works better[21]. LDA is also called the Fisherface method (13). The LDA is considered as a better option than PCA and the main aim of LDA is to find a base of vectors which provides best discrimination among classes[2].

Hidayat et al. [22] in 2011 carried out a comparative study between PCA and LDA for feature extraction in face recognition on various datasets such as FACES94, FACES95, FACES96, JAFFE, etc. Results show that PCA outsmarts LDA where time is the constraint whereas LDA(FACES94- 99.90\%, FACES95- 90.80\%, FACES9697.20\%, JAFFE- 100.00\%) performed better than PCA(FACES94- 99.90\%, FACES95- 87.00\%, FACES96$94.00 \%$, JAFFE- 92.60\%) when recognition rate is to be considered. Then Tomesh et al. [17]in 2013 combined PCA with LDA where former was used for dimension reduction whereas latter extracted the features. This combination on ORL face database gave $96.35 \%$ recognition rate beating various previously adopted methods. Bala et al. [23]in 2016 combined LDA(feature extraction) and Euclidean Distance classifier(for classification). Experiments carried over ORL database consisting 400 images of 40 people having 10 different poses each showed a recognition rate of $93.70 \%$.

\subsection{Discrete Cosine Transform (DCT)}

DCT is one of the most popular techniques of signal as well as image processing. It was initially introduced in early 70 s. Once the DCT is enforced on input image, the DCT coefficients are followed in zig-zag manner in order to convert image matrix into feature vector. Only a few DCT coefficients are enough for reducing redundancy and recovering original image from the selected coefficients[24].

Azam et al. [25] in 2010 presented a method of face recognition in which DCT was used for feature extraction and Artificial Neural Network(ANN) was used for recognition purpose. ORL, YALE and FERET databases were used for carrying out experiments where the result of the proposed method gave $98.01 \%$ recognition rate on ORL database and $92.77 \%$ on YALE database whereas in 2012 a combination of DCT for feature extraction and Nearest Neighbor Discriminant Analysis for face recognition by Tyagi et al.[26]. This method outperformed the one presented in [25] and gives a relatively high recognition rate of $99 \%$ (on ORL database) and $98.50 \%$ (over Yale database). In 2017 Preeti et al. [18]applied DCT-PCA+ CS and LDA+CS on FR System over ORL and Yale databases. The results proved the efficiency of proposed method over existing PCA and LDA.

\section{Feature Selection}

After feature extraction, the most important phase in face recognition process is the 'feature selection' phase. In Feature Selection phase, the most appropriate features that are needed by anFR system to deduce the desired results 
are selected. In other words, we can say that an FR System works on the "Garbage in Garbage Out" strategy. The key steps of the feature selection process are shown in Figure 2.

Subset Genesis is a heuristic lookup in which each state defines a test subset for evaluation. The nature of subset genesis procedure is determined by: successor generation and search organization. The new generated subset is then evaluated by some evaluation criteria to determine the goodness of generated candidate subset. Hence, a stopping criteria has to be determined to stop the selection process. At last, the generated subset is validated in the validation phase by carrying out various comparisons and tests with already established results. The three general approaches to feature selection are: Filter Approach, Wrapper Approach and Embedded Approach.Filter Approach selects the feature subset on the basis of scores of features in different statistical test instead of any learning algorithm. These methods are generally used as a pre-processing step. Wrapper Approach[27]uses a learning algorithm for evaluating generated subset and depending upon the results of previous model, features are added or removed from the feature subset. Embedded Approach[28]is a combination of both the Filter Approach and the Wrapper Approach. Using embedded approach methods, the feature subset are generated using the algorithms having their own built-in feature selection methods[29].

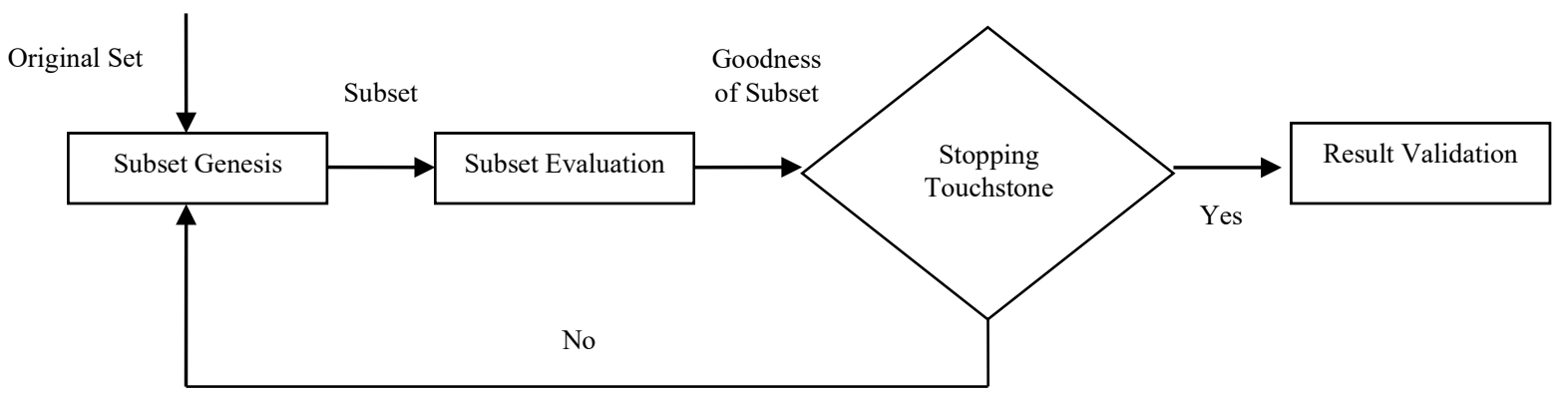

Figure 2: Key steps involved in Feature Selection Process(29)

7 Various optimization techniques present in literature such as Bat Algorithm[30], Particle Swarm Optimization (PSO)[31], Genetic Algorithm (GA)[32], Cuckoo Search Optimization Algorithm (CS)[33][34], Ant Colony Optimization (ACO)[35][36],Salp Swarm Algorithm (SSA)v[37] etc. helps in selecting features in a way that FRSystem gives as much accurate results as possible. Feature selection finds its application in fields like Text Categorization, Intrusion Detection, Image Retrieval, Remote Sensing etc.[29].

As we know PCA is used for extracting features and Memetic Algorithm is used for selecting features, Dinesh et al. [38]in 2009 combined both the strategies and applied PCA-MA approach on feature selection. The proposed approach clearly and completely outperformed the eigenface method as well as PCA led Genetic Algorithm (PCAGA). The eigenface method with 30 features produced $97.14 \%$ recognition rate whereas PCA-MA produced $100 \%$ recognition rate with just 17 features. Despite PCA being the widely used and easiest algorithm it has some limitations as well such as large computational load and poor discriminatory power. To overcome these limitations Manisha et al. [32] in 2014 proposed a fresh face recognition method based on the combination of PCA, Wavelet and SVM where low frequency component of wavelet were used for PCA representation, features were selected 
using Genetic Algorithm (GA) and Support Vector Machine along with nearest neighbor classifier (ND) was used for classification purpose. Experiments conducted on various databases such as ORL, FERET, Yale and YaleBproved the superiority of proposed method (ORL- 98.00\%, FERET- 97.30\%, Yale- $100.00 \%$, YaleB$100.00 \%$ ) over other leading methods. Preeti et al. [30] in 2017 showed an FR System using combination of DCT and PCA for dimension reduction and feature extraction succeeded by Bat Algorithm to select the best feature set. Experimental results proved the superiority of proposed method (97.5\% recognition rate with just 24 features) over other leading optimization techniques such as CS (97\% with 48 features), GA(96.5\% with 28 features) and PSO (96.5\% with 28 features). They further carried forward their research in 2017 in whichPreeti et al. [33]suggested anFR system using the Cuckoo Search (CS) optimization technique where features were extracted using combination of PCA and DCT in order to achieve high accuracy rates. Results show that CS gave $88 \%$ recognition rate with 10 features only which are equivalent to just $10 \%$ of the features used in PCA and gave 96.50\% recognition rate with just 34 features which is approximately $35 \%$ of the features used in DCT-PCA approach. Moving further Preeti et al. [39] in 2018studied effects of various distance classifiers on performance of anFR System over ORL database. Features were extracted using DCT-PCA and LDA and further selection was done using Cuckoo Search Algorithm. Among various distance classifiers Euclidean distance classifier produced the utmost93\% recognition rate followed by Manhattan Distance-92\%, Chebyshev Distance-89\% and Canberra Distance- $83 \%$ in absence of motion blur whereas in presence of motion blur results were: Euclidean Distance Classifier-91\%, Manhattan Distance-86\%, Chebyshev Distance- 83\% and Canberra Distance generated 74\% recognition rate. In 2019 Preeti et al. [40] further studied the effects of various distance classifiers on the performance of FR system based on Bat and CS algorithm over Yale_Original and Yale_Extended databases and results proved the supremacy of DCTPCA + Bat over PCA and DCTPCA + CS algorithms[41][42].

\section{Observations}

The following observations have been summarized from the literature review and computed in a tabular form.

Table 1: Results summarized from literature survey.

\begin{tabular}{|c|c|c|c|}
\hline $\begin{array}{c}\text { Reference } \\
\text { Paper }\end{array}$ & Technique Used & Database & Recognition Rate \\
\hline (14) & $\begin{array}{l}\text { Ada-Boosted Gabor Features } \\
\text { Resampling Technique }\end{array}$ & FERET & $\begin{array}{l}37.50 \% \text { (6 features) to } \\
95.20 \% \text { (700 features }) \\
90.60 \% \text { (700 features }) \text { to } \\
92.80 \% \text { (1740 features })\end{array}$ \\
\hline (15) & $\begin{array}{l}\text { PCA } \\
\text { 2DPCA } \\
\text { DiaPCA }\end{array}$ & FERET & $\begin{array}{l}85.50 \% \\
85.50 \% \\
90.50 \%\end{array}$ \\
\hline
\end{tabular}




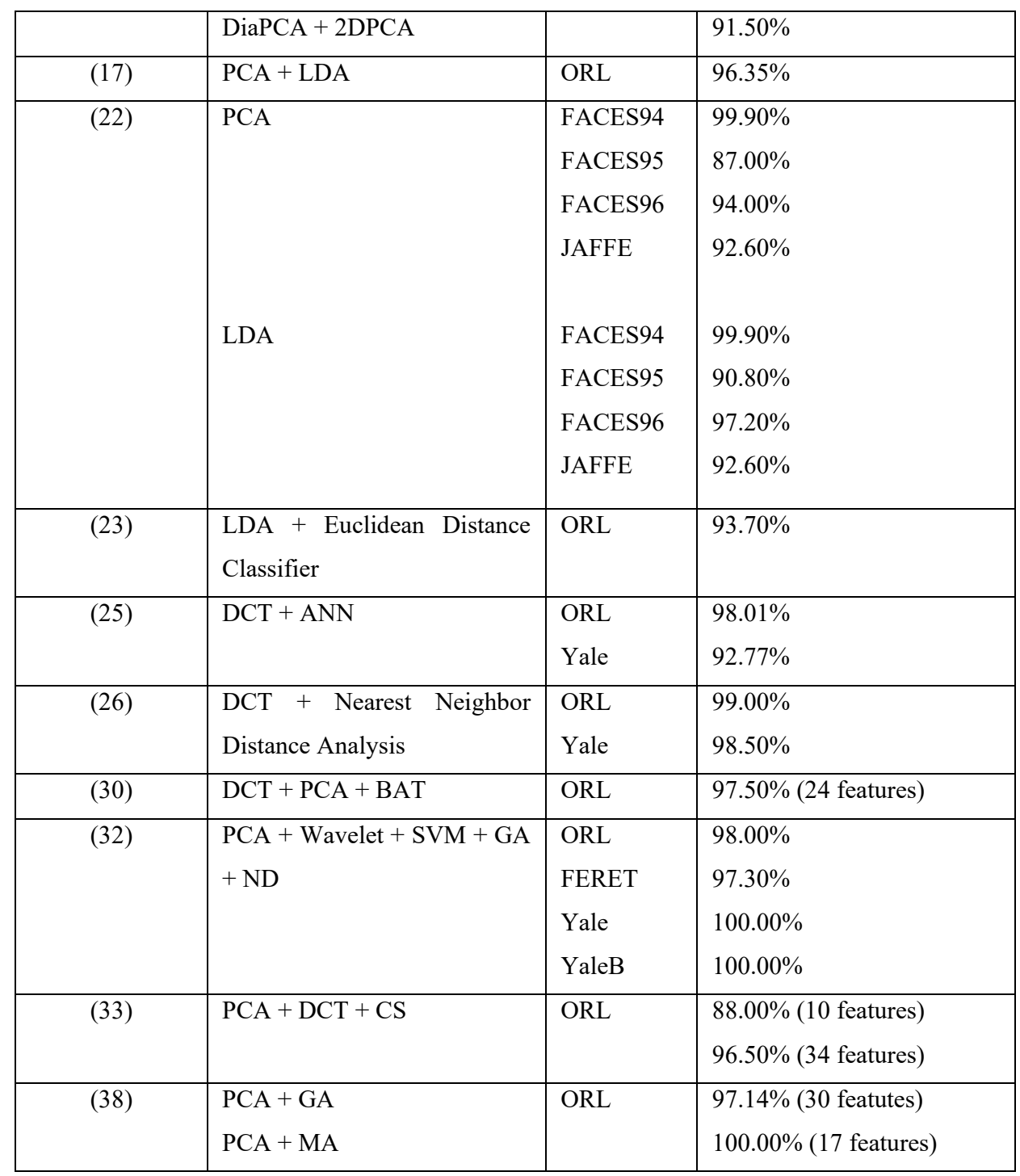

\section{Conclusion}

This paper seeks to go through considerable number of research and review papers in order to cover developments made till date in the field of Face Recognition. Face Recognition has been an attractive field for researchers from past few decades and is still the most favored topic for research owing to its vast practical applications. In this paper, we presented an extensive review of significant work done in the field of feature extraction and feature selection as well. The main challenges for face recognition mainly include pose variation, illumination, expressions and occlusion. Some of the most important techniques such as PCA, LDA, DCT, Bat Algorithm, Cuckoo Search Algorithm, etc. are explained well in this paper.The results obtained by various researchers using numerous different techniques are also compiled together in a tabular form in this paper. The objective of this literature survey is to help the potential researchers in the field of face recognition to understand the various methods and techniques present and to support their further researches. 


\section{References}

1. Hiremath P, Hiremath M. 3D Face Recognition based on Radon Transform, PCA, LDA using KNN and SVM. Int J Image Graph Signal Process. 2014 Jun 8;6:36-43.

2. Jain C, Sahu RK. A Survey: Face Recognition Techniques. 2014;5(1):8.

3. Kumar S, Singh S, Kumar J. A study on face recognition techniques with age and gender classification. In: 2017 International Conference on Computing, Communication and Automation (ICCCA). 2017. p. 1001-6.

4. wei-lun chao. face recognition. In.

5. Devadethan S, Titus G, Purushothaman S. Face detection and facial feature extraction based on a fusion of knowledge based method and morphological image processing. In: 2014 Annual International Conference on Emerging Research Areas: Magnetics, Machines and Drives (AICERA/iCMMD). 2014. p. 1-5.

6. Kamel MS, Shen HC, Wong AKC, Hong TM, Campeanu RI. Face recognition using perspective invariant features. Pattern Recognit Lett. 1994 Sep 1;15(9):877-83.

7. Wang J, Yang H. Face Detection Based on Template Matching and 2DPCA Algorithm. In: 2008 Congress on Image and Signal Processing. 2008. p. 575-9.

8. Molder C, Oancea R. Appearance-based facial detection for recognition. In: 2012 9th International Conference on Communications (COMM). 2012. p. 119-22.

9. Zhao W, Chellappa R, Phillips PJ, Rosenfeld A. Face recognition: A literature survey. ACM Comput Surv CSUR. 2003 Dec 1;35(4):399-458.

10. Kin-Man Lam, Hong Yan. An analytic-to-holistic approach for face recognition based on a single frontal view. IEEE Trans Pattern Anal Mach Intell. 1998 Jul;20(7):673-86.

11. Do TTT, Le TH. Facial Feature Extraction Using Geometric Feature and Independent Component Analysis. In: PKAW. 2008.

12. Saini R, Saini A, Agarwal D. Analysis of Different Face Recognition Algorithms. Int J Eng Res. 3(11):5.

13. Kaur R, Himanshi Er. Face recognition using Principal Component Analysis. In: 2015 IEEE International Advance Computing Conference (IACC) [Internet]. Banglore, India: IEEE; 2015 [cited 2020 Jan 24]. p. 585-9. Available from: http://ieeexplore.ieee.org/document/7154774/

14. Peng Yang, Shiguang Shan, Wen Gao, Li SZ, Dong Zhang. Face recognition using Ada-Boosted Gabor features. In: Sixth IEEE International Conference on Automatic Face and Gesture Recognition, 2004 Proceedings. 2004. p. 356-61.

15. Zhang D, Zhou Z-H, Chen S. Diagonal principal component analysis for face recognition. Pattern Recognit. 2006 Jan 31;39:140-2.

16. Delac K, Grgic M, Grgic S. Independent comparative study of PCA, ICA, and LDA on the FERET data set. Int J Imaging Syst Technol. 2005 Jan 1;15:252-60.

17. Verma T, Sahu R. PCA-LDA based face recognition system \& results comparison by various classification techniques. 2013 Int Conf Green High Perform Comput ICGHPC. 2013;1-7.

18. Preeti, Kumar D. Performance analysis of combination of CS with PCA and LDA for face recognition. In: 2017 International Conference on Information, Communication, Instrumentation and Control (ICICIC) [Internet]. Indore: IEEE; 2017 [cited 2020 Feb 28]. p. 1-6. Available from: http://ieeexplore.ieee.org/document/8279143/ 
19. Vinay A, Shekhar VinayS, Murthy KNB, Natarajan S. Face Recognition Using Gabor Wavelet Features with PCA and KPCA - A Comparative Study. Procedia Comput Sci. 2015 Jan 1;57:650-9.

20. Haitao Zhao, Pong Chi Yuen, Kwok JT. A novel incremental principal component analysis and its application for face recognition. IEEE Trans Syst Man Cybern Part B Cybern. 2006 Aug;36(4):873-86.

21. Pali V, Goswami S, Bhaiya LP. An Extensive Survey on Feature Extraction Techniques for Facial Image Processing. In: 2014 International Conference on Computational Intelligence and Communication Networks [Internet]. Bhopal, India: IEEE; 2014 [cited 2020 Jan 24]. p. 142-8. Available from: http://ieeexplore.ieee.org/document/7065462/

22. Hidayat E, Fajrian NA, Muda AK, Huoy CY, Ahmad S. A comparative study of feature extraction using PCA and LDA for face recognition. In: 2011 7th International Conference on Information Assurance and Security (IAS). 2011. p. 354-9.

23. Bala M, Singh P, Meena MS. FACE RECOGNITION USING LINEAR DISCRIMINANT ANALYSIS. 2016;4(2):8.

24. Sufyanu Z, Mohamad FS, Yusuf AA, Musa AN, Abdulkadir R. FEATURE EXTRACTION METHODS FOR FACE RECOGNITION. : 12 .

25. Azam M, Anjum MA, Javed MY. Discrete cosine transform (DCT) based face recognition in hexagonal images. In: 2010 The 2nd International Conference on Computer and Automation Engineering (ICCAE) [Internet]. Singapore: IEEE; 2010 [cited 2020 Feb 4]. p. 474-9. Available from: http://ieeexplore.ieee.org/document/5451613/

26. Tyagi SK. Face Recognition Using Discrete Cosine Transform and Nearest Neighbor Discriminant Analysis. Int J Eng Technol. 2012;4(3):311-4.

27. Vignolo L, Milone D, Behaine C, Scharcanski J. An evolutionary wrapper for feature selection in face recognition applications. In: 2012 IEEE International Conference on Systems, Man, and Cybernetics (SMC). 2012. p. 1286-90.

28. Hybrid feature selection by combining filters and wrappers | Request PDF [Internet]. ResearchGate. [cited 2020 Feb 28]. Available from:

https://www.researchgate.net/publication/220217148_Hybrid_feature_selection_by_combining_filters_and_wrappers

29. Kumar V. Feature Selection: A literature Review. Smart Comput Rev [Internet]. 2014 Jun 30 [cited 2020 Jan 25];4(3).

Available from: http://www.smartcr.org/view/download.php?filename=smartcr_vol4no3p7.pdf

30. Preeti, Kumar D. Feature selection for face recognition using DCT-PCA and Bat algorithm. Int J Inf Technol. 2017 Dec;9(4):411-23.

31. Ramadan RM. Face Recognition Using Particle Swarm Optimization-Based Selected Features. Image Process Pattern Recognit. 2009;2(2):15.

32. Dept of Electronics and Telecommunication Engineering, Sinhagad College of Engineering, Pune. 2Dean, Pune University, Pune., Satone M, Kharate G, Dept of Electronics and Telecommunication Engineering, Sinhagad College of Engineering, Pune. 2Dean, Pune University, Pune. Feature Selection Using Genetic Algorithm for Face Recognition Based on PCA, Wavelet and SVM. Int J Electr Eng Inform. 2014 Mar 30;6(1):39-52.

33. Malhotra P, Kumar D. An Optimized Face Recognition System Using Cuckoo Search. J Intell Syst. 2019 Apr 24;28(2):321-32.

34. (PDF) Wrapper Feature Selection using Discrete Cuckoo Optimization Algorithm [Internet]. [cited 2020 Feb 4]. Available from:

https://www.researchgate.net/publication/264310306_Wrapper_Feature_Selection_using_Discrete_Cuckoo_Optimization _Algorithm

35. Mafarja MM, Eleyan D. Ant colony optimization based feature selection in rough set theory. In 2013.

36. Kashef S, Nezamabadi-pour H. A new feature selection algorithm based on binary ant colony optimization. In: The 5th Conference on Information and Knowledge Technology. 2013. p. 50-4. 
37. Ahmed S, Mafarja M, Faris H, Aljarah I. Feature Selection Using Salp Swarm Algorithm with Chaos. In: Proceedings of the 2nd International Conference on Intelligent Systems, Metaheuristics \& Swarm Intelligence [Internet]. Phuket, Thailand: Association for Computing Machinery; 2018 [cited 2020 Feb 3]. p. 65-69. (ISMSI '18). Available from: https://doi.org/10.1145/3206185.3206198

38. Kumar D, Kumar S, Rai CS. Feature selection for face recognition: a memetic algorithmic approach. J Zhejiang Univ-Sci A. 2009 Aug 1;10(8):1140-52.

39. Preeti, Kumar D. Performance Evaluation of Face Recognition System using various Distance Classifiers. In: 2018 Second International Conference on Computing Methodologies and Communication (ICCMC) [Internet]. Erode: IEEE; 2018 [cited 2020 Jan 24]. p. 322-7. Available from: https://ieeexplore.ieee.org/document/8487835/

40. Preeti, Kumar D. Effect of Various Distance Classifiers on the Performance of Bat and CS-Based Face Recognition System. In: Yadav N, Yadav A, Bansal JC, Deep K, Kim JH, editors. Harmony Search and Nature Inspired Optimization Algorithms [Internet]. Singapore: Springer Singapore; 2019 [cited 2020 Jan 24]. p. 1209-20. Available from: http://link.springer.com/10.1007/978-981-13-0761-4_112

41. J. Dafni Rose*, K. Vijayakumar and S. Sakthivel, "Students' performance analysis system using cumulative predictor algorithm”, Int. J. Reasoning-based Intelligent Systems, Vol. 11, No. 2, 2019.

42. Vijayakumar. K, Nawaz Sherif. T, Gokulnath.S, “Automated Risk Identification using Glove algorithm in Cloud Based Development Environments", International Journal of Pure and Applied Mathematics Volume 117 No. 162017. 